\title{
RESISTENCIAS CIUDADANAS A LA MERCANTILIZACIÓN DEL PROCESO DE RECONSTRUCCIÓN Los sismos de 2017 en Ciudad de México e Ixtepec
}

\author{
Arnold, Pierre \\ (Investigador independiente) \\ pierre.arnold.pa@gmail.com \\ Ponce Arancibia, Natalia \\ (Candidata a Doctora en Urbanismo, Universidad Nacional Autónoma de México, becaria CONACYT) \\ natalia.ponce@gmail.com \\ Soto Delgado, Lisette \\ (Doctorante, Instituto de Geografía, Universidad Nacional Autónoma de México, becaria CONACYT) \\ lisette.sot.del@gmail.com
}

\section{RESUMEN}

Este artículo ofrece una aproximación a los efectos de los sismos del 7 y 19 de septiembre de 2017 en México, particularmente desde la política de reconstrucción del parque habitacional en Ciudad Ixtepec, estado de Oaxaca, y Ciudad de México. A pesar de las características espaciales y socioculturales disímiles entre ambos territorios se observa de manera transversal la implementación de estrategias de reconstrucción promotoras de esquemas de financiamiento que radicalizan el fenómeno de mercantilización de la vivienda. Los procesos de reconstrucción emprendidos a la fecha en estas ciudades permiten analizar las lógicas que han orientado la política habitacional y comprender cómo los desastres se transforman en oportunidades para el mercado inmobiliario y el sector de la construcción, sin importar el contexto socio-territorial en donde se implementan. Asimismo, este escenario permite observar las reacciones y resistencias de los damnificados frente a las opciones de reconstrucción de viviendas promovidas por el Estado.

Palabras clave: vivienda, sismos, mercantilización, resistencias, México

Bloque temático: Regeneración urbano-habitacional

\section{ABSTRACT}

This article offers an approximation to the effects of the earthquakes of September 7 and 19, 2017 in Mexico, particularly from the reconstruction policy of the housing stock in Ciudad Ixtepec, Oaxaca State, and Mexico City. Despite the dissimilar spatial and sociocultural characteristics of both territories, it is possible to observe in a transversal way the implementation of reconstruction, promoters of financing schemes that radicalize the phenomenon of commodification of housing. The reconstruction processes undertaken until now in these cities allow us to analyse the neoliberal logics in housing policy and understand how disasters are transformed into opportunities for the real estate market and the construction sector, regardless of the socio-territorial context where they are implement Likewise, this scenario allows to observe the reactions and resistances of the affected families in front of the housing reconstruction options promoted by the State.

Keywords: housing, earthquakes, commodification, resistances, Mexico

Topic: Urban-housing regeneration 


\section{Introducción}

La dimensión vivienda de manera recurrente es la que ha presentado mayores pérdidas a partir de diversos fenómenos sísmicos ocurridos en América Latina. Esta situación implica de manera inmediata el despojo de los lugares de residencia de miles de familias y se presenta a la vez como un potencial nicho para la ampliación de los intereses del sector inmobiliario.

A la luz de este enfoque, los efectos de los sismos ocurridos en 2017 en México podrían ser interpretados como episodios o hitos que profundizan aquellas políticas de vivienda trazadas previamente, convirtiéndose en muchos casos, en una oportunidad que perpetúa la producción habitacional dentro de un esquema donde prevalece la mercantilización de la vivienda. Cabe que señalar dicho fenómeno no es algo nuevo, este se ha construido a partir de un conjunto de reformas y políticas de liberalización económica que desde mediados de la década de los 80 se han promovido a nivel mundial, y que tienen un correlato en las estrategias urbanohabitacionales que se han implementando en la región (De Mattos, 2016). En este esquema también es importante precisar el rol que asume el Estado, quien ha transferido sistemáticamente gran parte de las funciones de planeación y gestión urbana al sector privado, condición que ha dado sustento a la lógica del capital inmobiliario en la producción y reproducción de la ciudad facilitando la actuación del sector inmobiliario y la industria de la construcción (De Carvalho y Corso-Pereira, 2013).

La lógica mercantil es la que prevalece en los esquemas de reconstrucción emprendidos en México, pudiendo afirmarse que el Estado se ha limitado a refrendar las decisiones del sector privado y crear las condiciones institucionales para su operación en el proceso (De Carvalho y Corso-Pereira, 2013), buscando generar rentabilidad por medio de bajos costos de operaciones, invalidando otras formas de producción no mercantil (Pírez, 2016), reproduciendo así grandes cantidades de viviendas de iguales características en todos los territorios (Arnold, 2018). Esta condición ha abierto un escenario tensión entre los damnificados y el Estado, pues los esquemas de financiamiento que tercerizan las acciones al sector privado, en desmedro de su calidad de vida, han suscitado el rechazo de las comunidades afectadas.

De esta manera, si bien el proceso de reconstrucción por un lado ha trazado una ruta que favorece la ampliación del capital inmobiliario, por otro ha abierto espacios de resistencia que demandan la modificación o propician nuevos esquemas para el abordaje de la rehabilitación o reconstrucción del parque habitacional afectado. En esta línea, Boaventura de Sousa Santos brinda una reflexión teórica que es útil para comprender la dinámica antes descrita, sugiriendo que existen dos tipos de soluciones para el abordaje de un problema común en un determinado contexto, estas son las soluciones institucionales y las soluciones extrainstitucionales. Las primeras surgen en el marco del sistema vigente y de las instituciones administrativas del Estado, operando al interior de ellas sin alterar su normal funcionamiento. Las segundas desafían el marco institucional existente, con el propósito de introducir transformaciones profundas o bien forzarlo a tomar medidas que de otro modo no tomaría (De Sousa Santos, 2014).

Con dicho cuestionamiento, y a partir de las experiencias en Ciudad México y Ciudad Ixtepec, nos aproximamos a las acciones de organización emprendidas por los damnificados, las que en alguna medida han confrontado un proceso de reconstrucción que entiende a la vivienda como mercancía, demandando mediante diversas formas sociales de valorización y apropiación, un proceso de reconstrucción que garantice la calidad de las viviendas y la permanencia en distintos enclaves de residencia afectados por el sismo. 


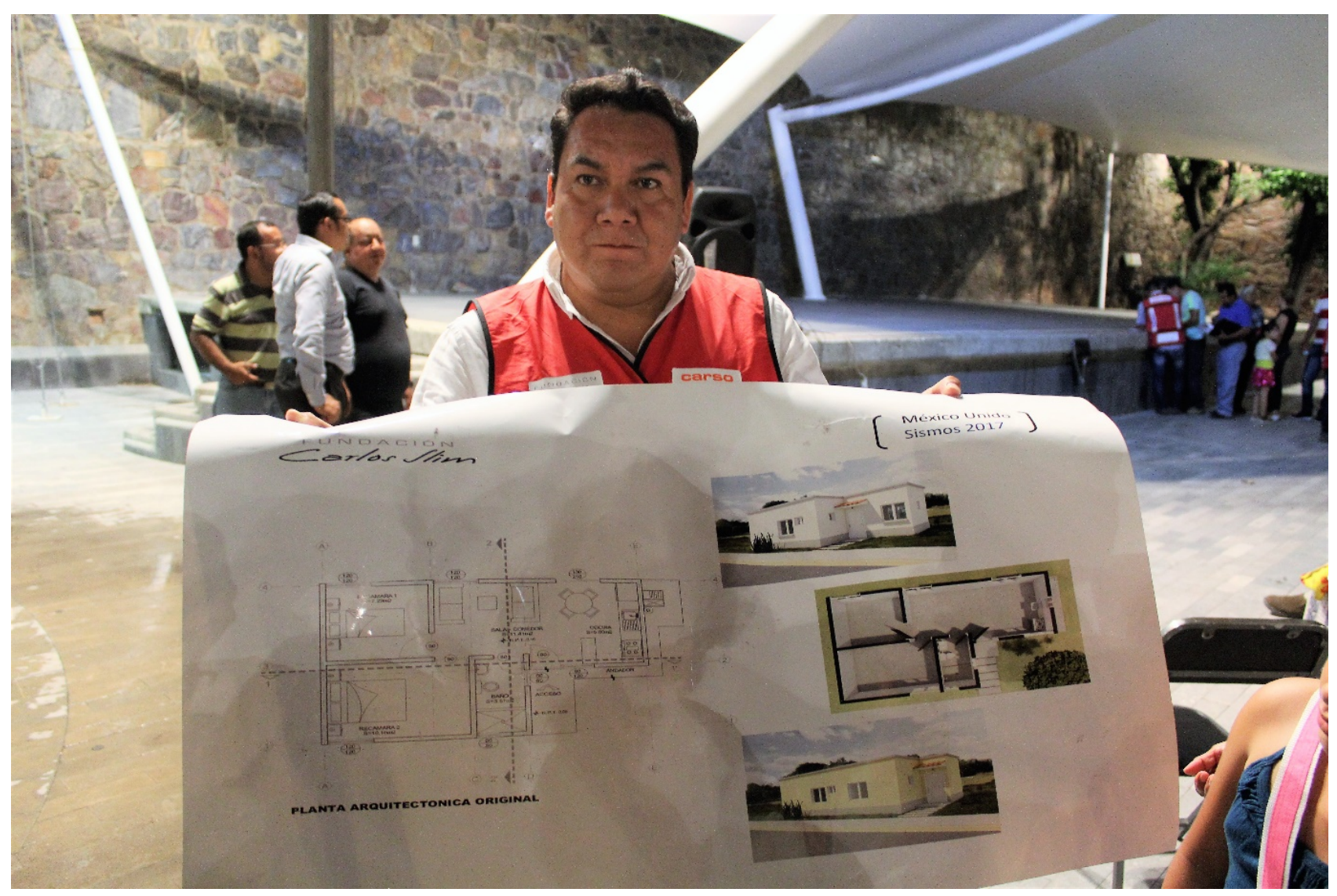

Fig. 1. Modelo de vivienda vendida a familias damnificadas por la Fundación Carlos Slim, Ciudad Ixtepec, Oaxaca, México. Fuente: Elaboración propia, 2017.

\section{Los habitantes frente a las opciones de reconstrucción en Ciudad Ixtepec, Istmo de Tehuantepec, Oaxaca}

\subsection{Septiembre de 2017, dos sismos, un censo en Ciudad Ixtepec}

Ciudad Ixtepec es un municipio emplazado en el sur-poniente de México en el Istmo de Tehuantepec, a 280 $\mathrm{km}$ de la capital regional, estado de Oaxaca. El 7 de septiembre dicho municipio se vio impactado por un fuerte sismo con epicentro en el Golfo de Tehuantepec que registró una magnitud de 8.2, con una réplica magnitud 6.1 el 23 de septiembre del mismo año (Servicio Sismológico Nacional, 2017). Producto del primer evento, fueron afectadas un total de 4130 viviendas, que corresponden aproximadamente a la mitad de las viviendas existentes en el lugar, convirtiéndose en uno de los tres municipios más afectados por el sismo en el estado de Oaxaca. Según datos del censo oficial realizado después del sismo (SEDATU, 2018), de las viviendas afectadas $42 \%$ tuvieron daño parcial y $58 \%$ total. Cabe señalar que el segundo sismo provocó nuevos daños a viviendas debilitadas por el sismo anterior y a viviendas que no habían sido afectadas, sin embargo no se llevó a cabo un nuevo censo para el catastro.

La tipología de vivienda que predomina en Ciudad Ixtepec es la vivienda unifamiliar con un 93,7 \% (INEGI, 2015). Su construcción se realiza mayoritariamente por medio de la autoproducción, es decir, son las mismas familias quienes intervienen en su diseño y edificación, bajo lógicas de producción no mercantil (Pírez, 2016), clasificadas en cuanto a materialidad y morfología dentro de lo que se denomina arquitectura tradicional o vernácula (De Leo, 2015) ${ }^{1}$. Sin embargo, en las últimas décadas -debido a la industria del cemento, el coste

${ }^{1}$ Viviendas con muros de tierra cruda (adobe o bahareque) o de ladrillos cocidos cubiertos de un repello con cal pintado, techos 
de los materiales y la mano de obra- existe un aumento de viviendas construidas con blocks de concreto y techos de lámina de zinc o asbesto. Pese a ello, la "vivienda tradicional istmeña", como la denominan sus habitantes, es la que posee mayor pertinencia y adaptación climática a las altas temperaturas, así como a las fuertes lluvias y vientos de la región.

\subsection{Deficiencias del proceso de reconstrucción de Ciudad Ixtepec}

La reconstrucción en el estado de Oaxaca se ha llevado a cabo por medio del Fondo de Desastres Naturales (FONDEN), el cual transfiere recursos económicos a estados afectados por desastres para la mitigación de daños en infraestructuras y equipamientos públicos, así como a familias de bajos ingresos para la reconstrucción o reparación de viviendas afectadas (Estrada, 2014). ${ }^{2}$ Para este estado, el gobierno federal liberó en total MXN 315525000 (USD 17355 610) para la reconstrucción, durante los meses posteriores a los sismos. Las indemnizaciones recibidas por los damnificados, previamente censados, por medio del FONDEN en Ciudad Ixtepec se estimaron en MXN 15000 (USD 825) en caso de haber sido censados con daño parcial y de MXN 120000 (USD 6 601) para daño total.

Por medio de este mecanismo económico se entregó a los damnificados por un lado, tarjetas de débito (tarjetas de pago del subsidio), y por otro, les fue proporcionado un servicio de asistencia técnica gratuita por medio de la contratación de los Organismos Ejecutores de Obras (OEOs) ${ }^{3}$, cuya principal labor sería asesorar y acompañar la autoproducción. En este sentido, el FONDEN fungió como primer requisito para que los damnificados pudieran acceder a una oferta de vivienda social, al mismo tiempo que se convirtió en complemento para el trabajo que desarrollan las empresas constructoras y OEOs en el territorio afectado, favoreciendo la consolidación del "paternariado público-privado" (De Mattos:42) mediante el traspaso de recursos públicos al sector de la construcción.

Una vez distribuidas las tarjetas de débito del FONDEN, las empresas constructoras que llegaron a Ciudad Ixtepec iniciaron una competencia por captar la demanda, incitando la demolición de viviendas tradicionales para ofertar nuevas viviendas "llave en mano" a las familias con daño total, todo esto amparado por un escenario poco claro respecto de los procedimientos esbozados para la reconstrucción. Lo anterior refuerza el paradigma de las políticas habitacionales neoliberales que promueven esquemas de producción de vivienda de interés social basadas en la estandarización, sin adaptaciones al contexto, a las familias ni a las condiciones climáticas (Arnold, 2018).

Los damnificados entraron entonces en una lógica en la cual podían escoger comprar a quienes les aseguraban tener una mejor vivienda en el menor tiempo posible, coyuntura que fue aprovechada por las empresas constructoras que buscaban ofertar en un breve periodo un gran paquete de viviendas estandarizadas. En este sentido, otro tipo de medidas como la reparación de viviendas existentes, la propuesta de modelos de viviendas adaptados a los terrenos y a las necesidades de las personas, o la reutilización de materiales recuperados, fueron inicialmente desatendidas. En su gran mayoría, la oferta de estas empresas ha permanecido inmutable a una vivienda básica con una sola planta de entre 40 y $55 \mathrm{~m}^{2}$ en promedio para dos habitaciones, sala, cocina y baño de tamaños muy reducidos, con alturas de techo por lo general inferiores a $2.70 \mathrm{~m}$, y con materiales económicos (paredes de block o de concreto), como las promovidas por el "tren de la vivienda" mexicana

con estructura de madera cubierta de tejas de barro. Espacialmente, poseen grandes superficies con uno o dos cuartos de entre 60 a $125 \mathrm{~m}^{2}$ y alturas de cuatro a seis metros adaptadas a las altas temperaturas de la región, así como un corredor cubierto y cocina y baño en el patio.

${ }^{2}$ Instrumento financiero que tiene por objetivo atender la recuperación efectos de desastres naturales cuando superan la capacidad de respuesta y de financiamiento de las autoridades locales. El apoyo de reconstrucción de vivienda mediante el FONDEN está sujeto a que los hogares damnificados demuestren habitar la vivienda permanentemente, estar en pobreza patrimonial, ser propietarios y que la tenencia del terreno sea regular.

${ }^{3}$ Según CONAVI al año 2018, existía un registro de 109 constructoras, inmobiliarias y asociaciones civiles inscritas en el padrón de OEO (CONAVI, 2018). 
señalado por Arnold (2018). Algunas empresas proponían viviendas más grandes con la posibilidad de contratar un crédito para cubrir el costo adicional al monto del FONDEN.

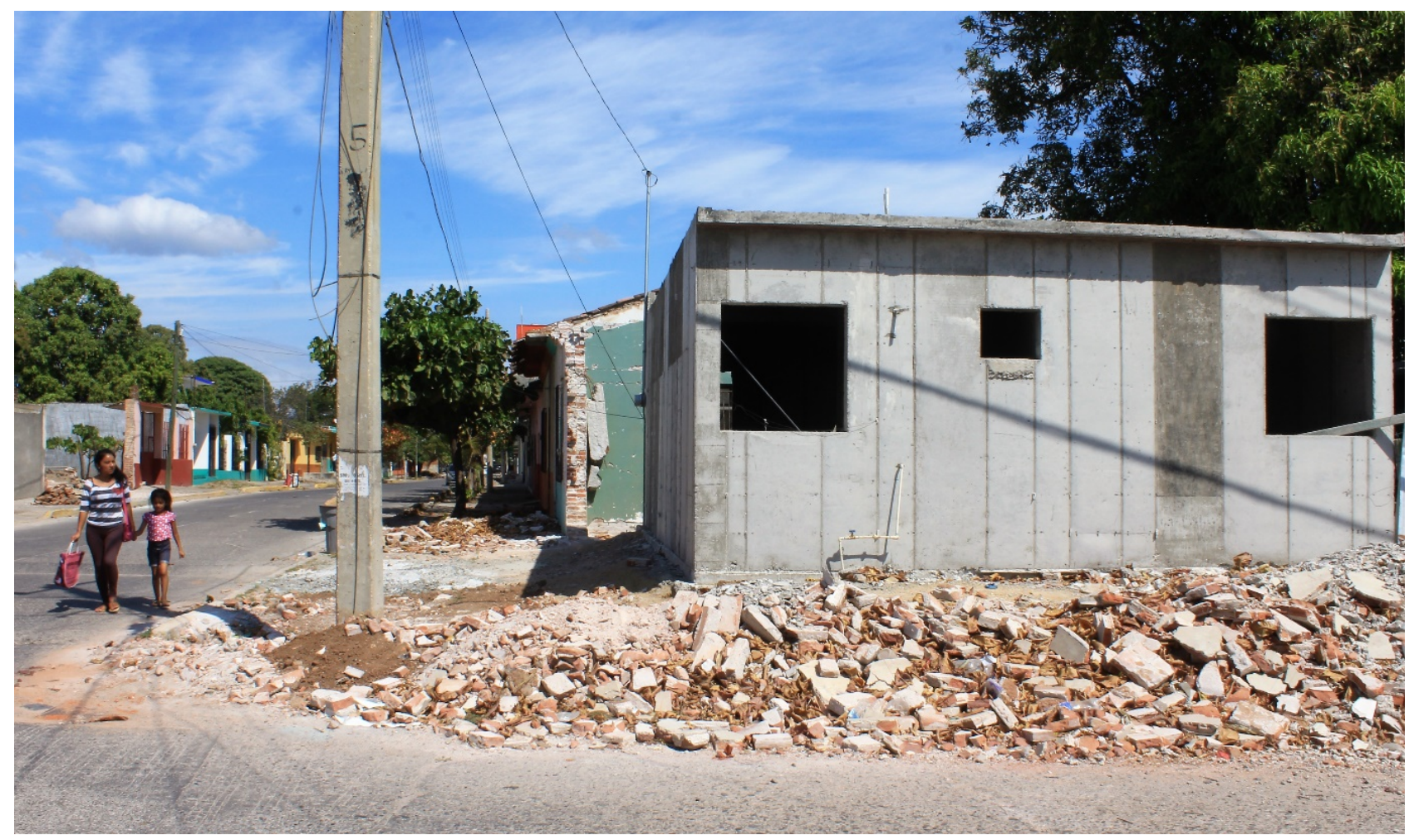

Fig. 2. Una vivienda tipo de constructora sustituye una casa tradicional, Ixtepec, Oaxaca. Fuente: Elaboración propia, 2018.

\subsection{La autoproducción y la restauración de la vivienda tradicional versus la vivienda "Ilave en mano"}

No obstante, la mayoría de las empresas que llegaron a Ciudad Ixtepec con estrategias basadas en satisfacer la necesidad de vivienda mediante esquemas estandarizados de producción, se retiraron sin haber podido capturar la demanda suficiente para aplicar su modelo de negocio, basado en una lógica mercantil (Pírez, 2016) que rentabiliza las ganancias por medio de bajos costos de operaciones (reducción de superficies y baja calidad de materiales). Este espacio fue abordado por un par de organizaciones sin fines lucrativos que apostaron por apoyar a las familias -mediante los recursos del FONDEN o fondos propios- para restaurar las viviendas tradicionales mediante la reutilización de materiales y de refuerzos que no vieran afectada la estructura original, o bien técnicas tradicionales y ecológicas en la reconstrucción de viviendas. Sin embargo, estas iniciativas que buscaron una mayor capacitación y participación de los habitantes en la reconstrucción tuvieron dificultades para llevar a cabo sus propuestas alternativas y convencer a las familias en un contexto post desastre.

Es interesante observar lo que ocurrió en Ciudad Ixtepec porque la principal vía elegida por los damnificados Ixtepecanos para la reconstrucción o reparación de sus viviendas fue la autoproducción, tanto por los que no fueron considerados por el censo como por los que recibieron la ayuda parcial o completa del FONDEN. Esta elección resulta relevante pues señala una tendencia que prioriza la producción de vivienda auto-producida de manera no mercantil, es decir, producida bajo los intereses y para el propio consumo de quienes las necesitan (Pírez, 2016). Entre los principales motivos de esta elección se pueden mencionar: la posibilidad que tienen los habitantes de reparar la vivienda familiar como parte de un patrimonio, la desconfianza hacia los actores que llegaron y el rechazo a los modelos de vivienda "llave en mano" inadaptados al contexto, así como la preferencia de controlar el proceso mediante la contratación directa de un albañil (local, llegado de otro estado o migrante) y desde el entendimiento de la construcción por parte de los damnificados o sus familiares. 
Si bien los damnificados de Ciudad Ixtepec no generaron instancias de organización colectiva a modo de demandas hacia la política de reconstrucción formal por parte del Estado, la mayoría hizo efectivo el principal mecanismo financiero impulsado por la política de reconstrucción, el FONDEN, porque implica un recurso que, en lo concreto, permite avanzar en la reconstrucción de la vivienda. En cuanto a la "asesoría técnica" por parte de las empresas OEOs, el tema de la construcción antisísmica ha sido relegado a un segundo plano frente a la necesidad de las familias por tener rápidamente una vivienda. Tanto las casas construidas por constructoras como las autoproducidas repiten los métodos constructivos anteriores al sismo.

En este sentido, el apoyo del gobierno se ha hecho cómplice de una reconstrucción caótica en la cual ha privilegiado la entrada al territorio de empresas constructoras y equipos técnicos, cuyo fin no siempre ha sido el de reconstruir una vivienda acorde a las necesidades de los habitantes damnificados, sino contrariamente, muy distantes en material y forma a lo requerido. A nuestro parecer esta situación tiene que ver, como ya se ha señalado, en el descanso de la reconstrucción en soluciones institucionales orientadas en mecanismos y esquemas neoliberales (Arnold, 2018; Pírez, 2016; De Mattos, 2016), que se traducen en "dejar hacer" a los habitantes como a las empresas con los subsidios liberados, al mismo tiempo que se realizan traspasos de recursos públicos al sector privado, se promueven esquemas de mercantilización de la vivienda por sobre los esquemas existentes no mercantiles, se introducen lógicas de competencia y consumo (oferta-demanda).

En cuanto a la solución efectiva, pensando en que para los habitantes el fin último -de manera práctica, sería el restablecimiento de la vivienda-, ésta se ha concretado por ellos mismos, de manera extrainstitucional y mucho más a corriente con sus prácticas históricas de autoproducción de la vivienda. En este caso vemos que el objetivo principal de los damnificados sería llegar a una reconstrucción concreta, real de la vivienda, más que interpelar al Estado junto a sus esquemas y estrategias desplegadas. La solución de los habitantes en la reconstrucción de la vivienda, quizá forzosamente llamada resistencia, no se realiza por rebeldía o por un deseo de transformación de la política institucional sino más bien por una necesidad práctica de retornar a una vivienda. 


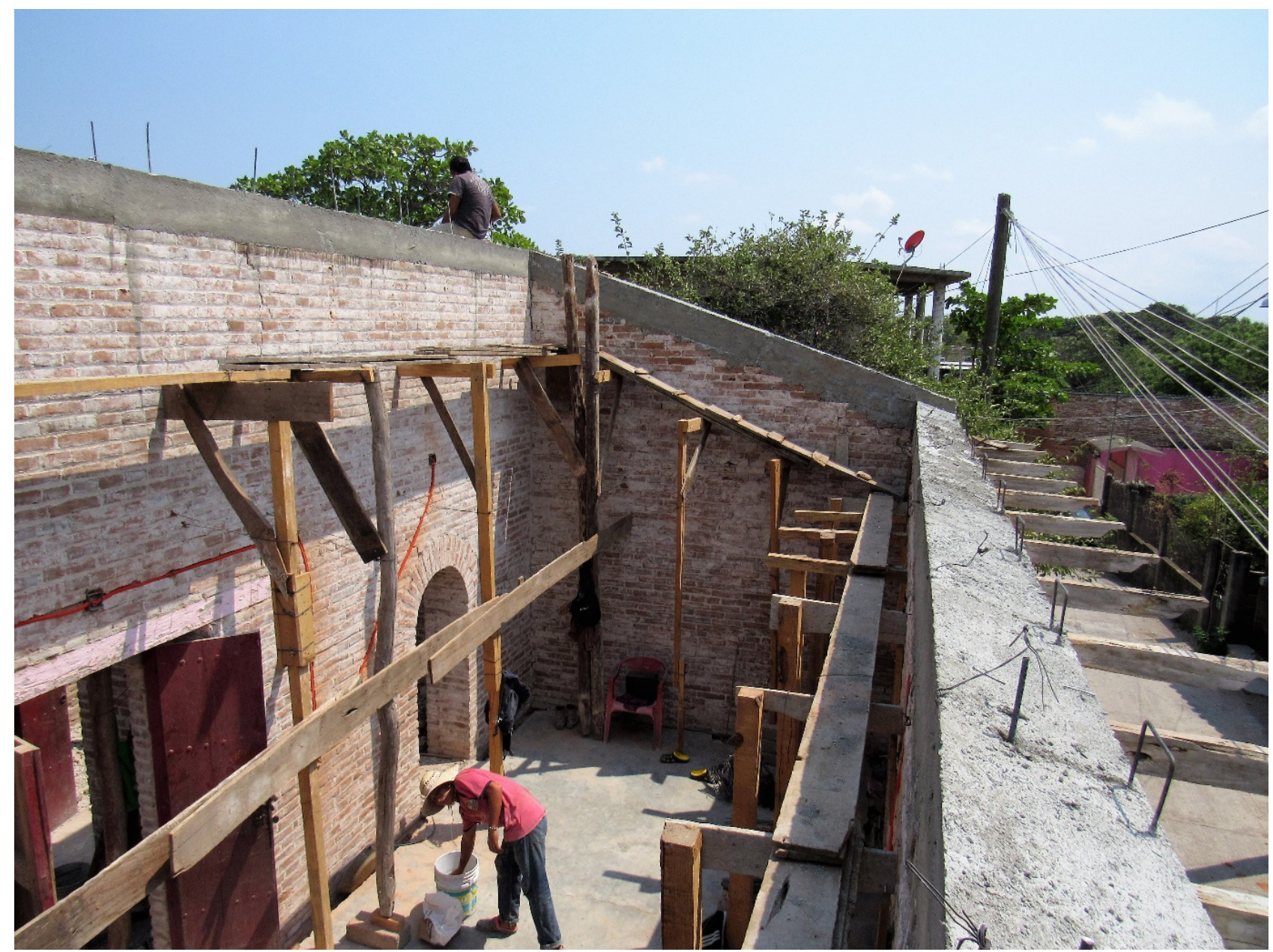

Fig. 3. Reparación y refuerzo antisísmico de una vivienda tradicional istmeña con la asesoría de una asociación civil mexicana, Ixtepec, Oaxaca. Fuente: Elaboración propia, 2018.

\section{Resistencias a un modelo impuesto: acciones ciudadanas y la reconfiguración de los esquemas de reconstrucción en la Ciudad de México.}

\subsection{Antecedentes generales del sismo del 19 de septiembre de 2017}

El sismo de magnitud 7.1 que afectó a la Ciudad de México el 19 de septiembre de 2017 tuvo su epicentro a $120 \mathrm{~km}$ al sur de la capital (UNAM, 2017) provocando afectaciones diferenciadas en distintos puntos de la ciudad, donde los mayores daños recayeron sobre inmuebles de uso habitacional. ${ }^{4}$ En este contexto, se observan diferencias en el patrón de tipologías habitacionales afectadas y su distribución territorial: por un lado, los daños asociados a viviendas unifamiliares se ubican preferentemente en alcaldías del primer contorno Tláhuac, Iztapalapa y Xochimilco- y por otro, las afectaciones asociadas a edificios de vivienda multifamiliar se asocian principalmente a las alcaldías centrales de Cuauhtémoc, Benito Juárez y Coyoacán.

De manera complementaria cabe señalar que el reciente fenómeno afectó de manera transversal a distintos sectores socioeconómicos, incluyendo de manera significativa a sectores medios y medios altos residentes de colonias emplazadas en alcaldías centrales, gran parte de ellos residentes de edificios de vivienda multifamiliar. En este sentido, el estudio de dichas delimitaciones territoriales se torna relevante por la a la rentabilidad del suelo en el cual se emplazan los inmuebles, evidenciándose en ellas de manera más directa la instalación de

${ }^{4}$ De los 2626.672 registros informados por la Comisión para la Reconstrucción de la Ciudad de México (2018), 25.831 corresponden a vivienda unifamiliar o edificios multifamiliares. 
procesos especulativos, facilitados además por la estructura institucional montada para atender el proceso de reconstrucción desde el Gobierno de la Ciudad.

\subsection{La mercantilización del proceso de reconstrucción de la Ciudad de México}

El fenómeno sísmico en cuestión puso en evidencia la vulnerabilidad territorial que caracteriza a la Ciudad de México, condición que se expresa en la compresibilidad del suelo (que favorece la amplificación de las ondas sísmicas), su organización espacial y las características demográficas propias de la capital. Pero de manera más relevante enfatizó diversas falencias en la estructura institucional, que han generado conflictividades extendidas desde la etapa de emergencia hasta el proceso de reconstrucción. Cabe señalar que esta condición obedece de manera estructural al desmantelamiento experimentado sostenidamente por el Estado mexicano en el marco del modelo de desarrollo neoliberal (De Mattos, 2016). Específicamente en el contexto de la reconstrucción, dichas falencias se expresaron de manera inicial en la ausencia de acciones concretas que complejizaron el abordaje de la situación de emergencia que enfrentaron miles de ciudadanos. No obstante es la etapa de reconstrucción la que presenta un aprovechamiento coyuntural más evidente para la radicalización de ciertas lógicas mercantiles, pues los instrumentos diseñados, como se señala a continuación, de manera expresa externalizan y favorecen las condiciones para la actuación del capital inmobiliario, enfatizando la retracción del Estado en el proceso de atención del parque habitacional afectado.

Así, el abordaje de los daños experimentados por los edificios de vivienda multifamiliar emplazados en las alcaldías centrales, supone un análisis que parte de las lógicas mercantiles que matizan el actual contexto de producción habitacional en la Ciudad de México. En este sentido el patrón de distribución territorial de los multifamiliares afectados se presenta como un factor relevante, pues se condice con sectores donde el precio de la tierra es más elevado. Por tanto, retomando las reflexiones de De Mattos (2016: 45) (2016), es posible advertir un interés particular de promotores inmobiliarios por estos territorios debido a "la existencia de una mayor demanda solvente, tanto para vivienda como para servicios", siendo potenciales espacios para nuevas inversiones inmobiliarias. En este contexto, la acción institucional orientada de manera inicial a la rehabilitación o reconstrucción de edificios de vivienda multifamiliar se canalizó por el "Crédito para la reparación estructural de vivienda vertical" a través de la Sociedad Hipotecaria Federal (SHF) y también por lo dispuesto en la Ley para la Reconstrucción de la Ciudad de México. Ambas medidas se sostuvieron por al menos un lapso de 7 meses como únicas alternativas a las que podían acceder los damnificados de multifamiliares de vivienda.

De manera sintética podemos señalar que el primer instrumento contempla explícitamente la disposición de créditos para los damnificados; el segundo propone mecanismos en una línea similar, donde la estrategia de redensificación, orientada a aquellos edificios con daños estructurales irreparables que deben ser demolidos y reconstruidos, funge como un mecanismo orientado a la rentabilización del suelo. En términos concretos, dicho mecanismo consiste en el aumento de la constructibilidad de los predios hasta en un $35 \%$, expresado en la edificación de niveles adicionales a los que originalmente poseía el edificio. Adicionalmente, los propietarios deben gestionar un crédito para dar inicio a las obras, el cual supuestamente sería pagado con la venta de las unidades de viviendas obtenidas a partir del aumento de constructibilidad. Con los antecedentes antes expuestos, no es arriesgado pensar que el empleo de estrategias como la redensificación, más que atender la necesidad de vivienda de los damnificados, busca propiciar condiciones que impulsen nuevos negocios inmobiliarios. Estas acciones sin duda buscan dar continuidad e intensificar la transformación y mercantilización del desarrollo urbano que se ha venido dando en las alcaldías centrales, enfatizando la tendencia de que promueve la competitividad urbana como forma de crecimiento de la ciudad, mediante el empleo de mecanismos de mercado como los antes descritos (De Carvalho Y Corso-Pereira, 2013).

\subsection{Resistencia colectiva a las estrategias de reconstrucción: Damnificados Unidos de la Ciudad de México.}

No obstante, la ruta institucional diseñada para la reconstrucción ha marcado un punto de inflexión, siendo rechazada por gran parte de los afectados, quienes han insistido sistemáticamente no estar dispuestos a asumir la condición de deudores cuando lo realmente crítico es ser damnificados, ni acceder a esquemas 
financieros que emplean la rentabilización del suelo mediante la introducción de estrategias como la redensificación.

En este sentido, el movimiento social gestado en torno al proceso de reconstrucción en la Ciudad de México responde de manera inicial a la ausencia manifestada por el estado una vez ocurrido el sismo y ha sostenido su resistencia frente a las lógicas de corte neoliberal esbozadas de manera posterior a la promulgación de la Ley de Reconstrucción para la Ciudad de México. Cabe señalar que al hablar de movimiento social se reconocen según Melucci (1990:362)(1990) al menos dos elementos fundamentales en su configuración, uno relacionado con la acción colectiva y la capacidad de los actores para reconocerse en una misma unidad social y otro vinculado a la presencia de un conflicto, entendido este como "una situación en la cual dos adversarios se encuentran en oposición sobre un objeto común, en un campo disputado por ambos" .

En este contexto, las primeras acciones colectivas relacionadas con los damnificados tienen su origen en el Multifamiliar Tlalpan, predio ubicado en el sector nororiente de la alcaldía de Coyoacán, reconocido por el desplome de uno de sus bloques al momento del sismo donde perdieron la vida 9 de sus habitantes. Es desde este enclave donde se da inicio a un intenso proceso de articulación y difusión mediante asambleas y comunicados de prensa que buscaron posicionar las demandas de los damnificados en la opinión pública. En semanas posteriores dichas acciones se extrapolan a otros predios afectados, comenzando a gestarse un "sujeto colectivo que no solo se entendía a sí mismo como una agrupación de damnificados, sino como actores políticos que luchan contra el incumplimiento y la falta de voluntad del Estado para atender puntualmente las necesidades de las y los afectados" (Guerrero, 2018), conformándose así el día 18 de noviembre de 2017 el Colectivo Damnificados Unidos de la Ciudad de México. La colectivización de la demanda de los damnificados a través de dicha agrupación ha logrado incidir en la modificación de algunos aspectos operativos relevantes vinculados a la Ley de Reconstrucción.

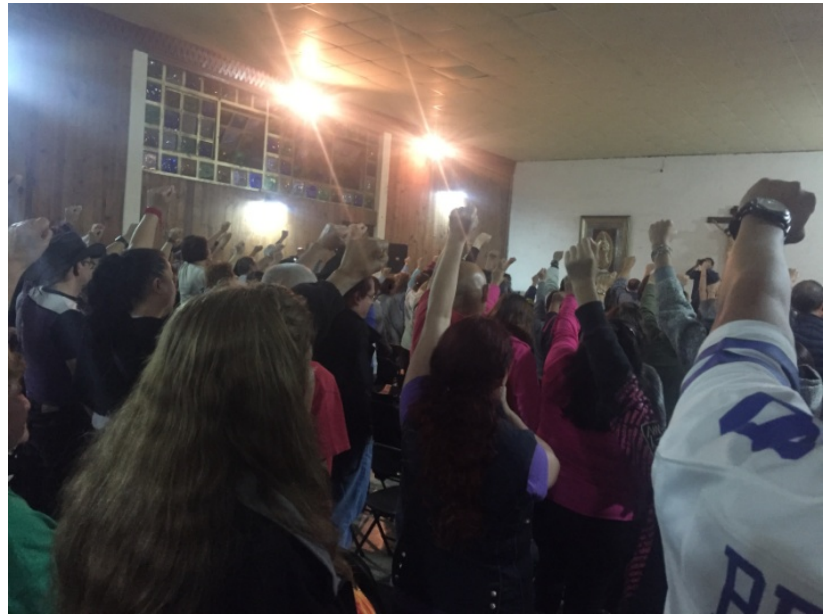

Fig. 4. Asamblea en el Multifamiliar Tlalpan Fuente: Elaboración propia, 2018.

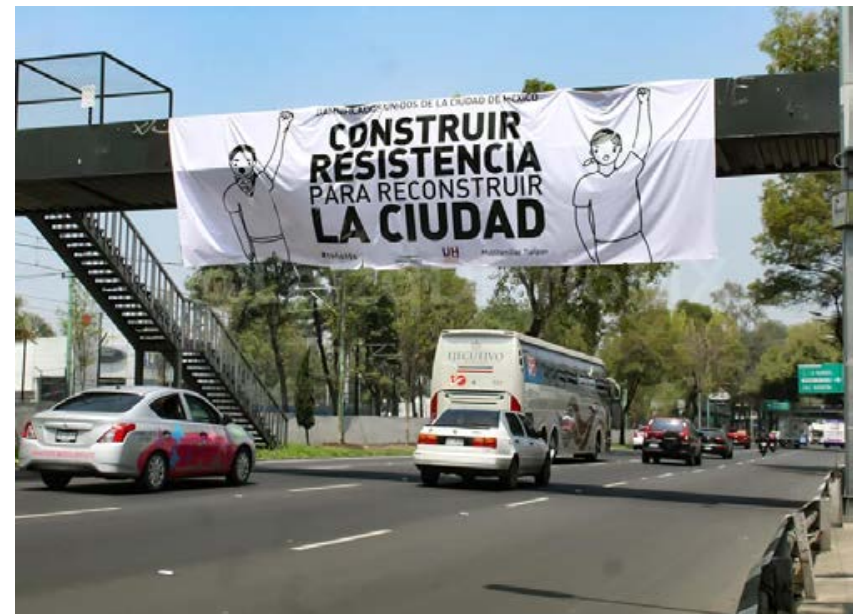

Fig. 5. Lienzo conmemorativo 19S Fuente: La Izquierda Diario México, 2018.

Damnificados Unidos ha conseguido posicionar un conjunto de demandas ante las autoridades e instituciones que intervienen en el proceso de reconstrucción de la ciudad mediante mesas de trabajo periódicas, las que, entre otras acciones, han posibilitado la construcción de una ruta de financiamiento con cargo a fondos públicos. En esta ruta rescatamos dos hitos relevantes: el primero, acontecido el 1 de mayo de 2018, se relaciona con la apertura al dialogo por parte las autoridades en lo relativo a la incorporación de fondos públicos para el financiamiento de obras de rehabilitación de edificios, así como a la revisión de la redensificación como estrategia de reconstrucción. El segundo tiene lugar en junio de 2018, mes en el que de manera extraordinaria la Comisión de Gobierno de la Asamblea Legislativa del Distrito Federal (ALDF), sesiona y aprueba el Fideicomiso Público para la Reconstrucción y Rehabilitación de Viviendas de Uso Habitacional y posteriormente expide los lineamientos para otorgar fondos para la reconstrucción y rehabilitación de viviendas 
(conjuntos habitacionales y edificios de uso habitacional multifamiliar) (Órgano de Difusión del Gobierno de la Ciudad de México, 2018a, 2018b).

Las medidas antes señaladas, han permitido a un conjunto de damnificados acceder a recursos públicos para la reconstrucción de sus inmuebles, sin créditos y sin redensificación como lo expresa la consigna levantada por el colectivo. De esta manera al finalizar la administración anterior, en noviembre de 2018, el total de subcuentas abiertas ascendía a 103 (Comisión para la Reconstrucción, 2018), vale decir, a esa fecha se habían incorporado 103 inmuebles al Fideicomiso Público para la reconstrucción. Es importante señalar que este esquema no habría tenido lugar sin la organización sostenida y el empleo diversas medidas de presión, necesarias ante la indiferencia e inflexibilidad de las autoridades capitalinas. La lucha emprendida por la organización en su etapa inicial sirvió para reivindicar el derecho de miles damnificados por permanecer en sus colonias y no perder sus viviendas por la imposibilidad de financiar su rehabilitación o reconstrucción, evitando así procesos de desplazamiento forzados. No obstante, los desafíos de Damnificados Unidos persisten y se relacionan por un lado con la presión para la dictaminación de los inmuebles que aún se encuentran en evaluación y por otro, con la fiscalización activa del inicio de obras de los predios que ya se encuentran incluidos en el Fideicomiso.

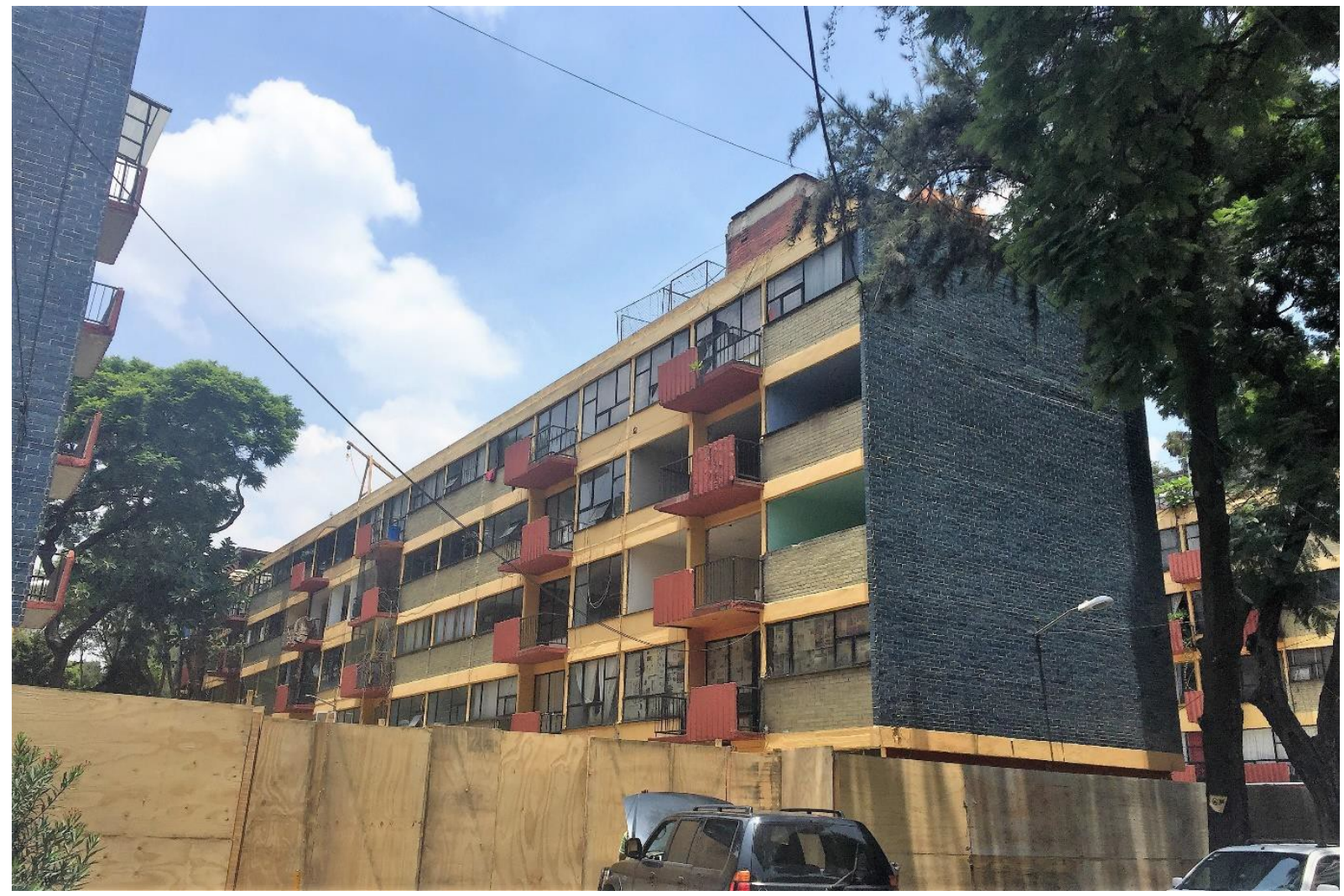

Fig. 6. Multifamiliar Tlalpan Fuente: Elaboración propia, 2018.

\section{Reflexiones finales}

Los casos revisados referencian los procesos de reconstrucción emprendidos tanto en Ciudad Ixtepec como en Ciudad de México y la resistencia que han presentado grupos de damnificados frente a la radicalización de esquemas mercantiles desarrollados en torno a la vivienda..

En Ciudad Ixtepec, la principal reconstrucción elegida por los habitantes por medio de la autoproducción de la vivienda así como de las pocas organizaciones especializadas en la restauración y refuerzo antisísmico de 
viviendas tradicionales, representan instancias que consideramos abren caminos interesantes hacia nuevas reconstrucciones posibles. En este sentido, se convierten en espacios propicios para repensar lo existente, para entender que la reconstrucción no se resuelve con soluciones de consumo, como es reforzada desde la lógica neoliberal; al mismo tiempo, que representa un espacio para profundizar esquemas de reconstrucción con nuevas lógicas, no mercantilizadas, y más cercanas a la dimensión política del habitante.

Por otro lado, el trabajo de Damnificados Unidos ha abierto una nueva ruta en el esquema de financiamiento para la reconstrucción de viviendas, que hoy se refleja en el reciente Plan Integral para la Reconstrucción de la Ciudad de México impulsado por la nueva autoridad capitalina, cuyos lineamientos establecen expresamente que no se convertirá a las personas damnificadas en deudores de la banca, ni se les coaccionará para el empleo de la estrategia de redensificación. Lo anterior mediante una vía de financiamiento única a través del Fideicomiso público en el caso de rehabilitación de edificios y de créditos puente a tasa 0 para aquellos que eventualmente opten por la redensificación de los predios, Estas declaraciones sintonizan con las principales demandas impulsadas por el colectivo. Este modo de operar nos demuestra una vez más que la acción ciudadana coordinada, mediante el empleo de estrategias extrainstitucionales, tiene la capacidad de desafiar la estructura institucional y forzarla a tomar medidas que en otras circunstancias difícilmente tomaría (Santos de Sousa, 2014: 39-40).

Finalmente, si bien los contextos de desastres y los procesos de reconstrucción son potenciales catalizadores de esquemas mercantiles de vivienda, por la necesidad de agilizar la producción para la recuperación de del parque habitacional afectado, simultáneamente poseen la capacidad de generar un espacio para la construcción de ciudadanía y organización que contravengan imposiciones que van en desmedro de la ocupación justa del territorio. Sin embargo este espacio de oportunidades presenta grandes desafíos, principalmente en lo referido a cómo capitalizar la experiencia de organización ciudadana surgida al alero de una contingencia (experiencias pos sismo), y lograr trasladarla a espacios más permanentes de luchas y resistencia en las ciudades, necesarios para garantizar derechos de permanencia y calidad de viviendas a nivel regional.

\section{Bibliografía}

ARNOLD, P. (2018). Políticas de producción y gestión social del hábitat en América Latina: conquistas de derechos e incidencia política frente a la "vivienda de interés social" orientada al mercado. En Salinas, Luis (coord.) "Gestión urbana y política de vivienda. Espacio público, (in)seguridad y conflicto urbano", Instituto de Geografía - UNAM y Editorial Monosílabo, pp 225-260. Artículo entregado para la publicación.

DE CARVALHO, I., y CORSO-PEREIRA, G. (2013). A cidade como negócio. EURE , 5-26.

DE LEO, A. (2015). Catálogo de Arquitectura Vernácula de Oaxaca. México: Secretaría de las Culturas y Artes de Oaxaca.

DE MATTOS, C. (2016). Financiarización, valorización inmobiliaria. Sociologias, Porto Alegre 18(42), 24-52.

DE SOUSA SANTOS, B. (2014). Democracia al borde del caos. Ensayo contra la autoflagelación. Bogotá: Siglo XXI editores.

ESTRADA, G. (2014). Puesta en práctica de una política de desastres: los instrumentos de la gestión de riesgos en México. Bulletin de l'institute français d'études andines. 43(3) 611-632.

MELUCCI, A. (1990). La acción colectiva como construcción social. XII Congreso Mundial de Sociología, (pp. 357-364). Madrir.

PÍREZ, P. (2016). Buenos Aires: la urbanización neoliberal de la urbanización metropolitana. Sociologias, Porto Alegre 18(42) 90-118. 


\section{Fuentes electrónicas}

BANCO MUNDIAL (2009). World Bank lauches Multicat program. http://www.worldbank.org/en/news/press-release/2009/10/19/world-bank-launches-multicatprogram (Consulta: 29/11/2018)

$\begin{array}{lccrr}\text { CONAVI } & \text { (2018). } & \text { Padrón } & \text { OEO } & 2018 . \\ \text { https://www.gob.mx/cms/uploads/attachment/file/416855/Padron } & \text { OEO } & \text { 2018.pdf } & \text { (Consulta: }\end{array}$
27/11/2018).

Comisión para la Reconstrucción de la Ciudad de México (2018). Acuerdos y Actas del Comité Técnico del Fideicomiso para la Reconstrucción: Comisión para la Reconstrucción. https://plataforma.cdmx.gob.mx/presupuesto/fideicomiso (Consulta: 26/02/2019)

COORDINACIÓN NACIONAL DE PROTECCIÓN CIVIL (2014). Conoce el SINAPROC. http://www.proteccioncivil.gob.mx/en/ProteccionCivil/Conce el SINAPROC (Consulta: 18/06/2018).

Gobierno de la Ciudad de México (2019). Portal para la Reconstrucción. https://reconstruccion.cdmx.gob.mx/ (Consulta: 26/02/2019)

GUERRERO, L. (2018). La emergencia de un sujeto colectivo: el caso de damnificados unidos de la Ciudad de México: Documenta desde abajo. http://documentadesdeabajo.org/informe-G-organizaciones-01damnificados-unidos.html (Consulta: 26/02/2019)

INEGI (2015). México en cifras: Oaxaca. http://www.beta.inegi.org.mx/app/areasgeograficas/?ag=20\#tabMCcollapse-Indicadores $\quad$ (Consulta: 02/11/2018).

LÓPEZ, A. (2018). Más de 8 mil casas tradicionales se pierden en Juchitán. http://oaxaca.eluniversal.com.mx/municipios/10-09-2018/mas-de-8-mil-casas-tradicionales-se-perdieron-enjuchitan (Consulta: 10/09/2017).

Órgano de Difusión del Gobierno de la Ciudad de México (2018a). Decreto por el que se reforman y adicionan diversas disposiciones del reglamento de la Ley de Presupuesto y gasto eficiente del Distrito Federal.http://data.consejeria.cdmx.gob.mx/portal old/uploads/gacetas/3988215ffe8a7fd29350a18fa5626ab6. pdf (Consulta: 19/01/2019).

Órgano de Difusión del Gobierno de la Ciudad de México (2018b). Lineamientos para otorgar el apoyo en reconstrucción y rehabilitación de viviendas en conjuntos habitacionales y edificios de uso habitacional multifamiliar afectados por el fenómeno sísmico del 19 de septiembre de 2017. http://data.consejeria.cdmx.gob.mx/portal old/uploads/gacetas/e0333cc2157b9bb559dcef706e44b390.pdf (Consulta: 19/01/2019).

SEDATU. 2018. Censo de viviendas Dañadas por los sismos del mes de septiembre de 2017. http://transparencia.sedatu.gob.mx (Consulta: 02/11/2018).

UNAM (2017). ¿Qué ocurrió el 19 de septiembre de 2017 en México?: Ciencia UNAM. 28 de septiembre. http://ciencia.unam.mx/leer/652/-que-\%20ocurrio-el-19-de-septiembre-de-2017-enmexico- (Consulta: 20/10/2017). 